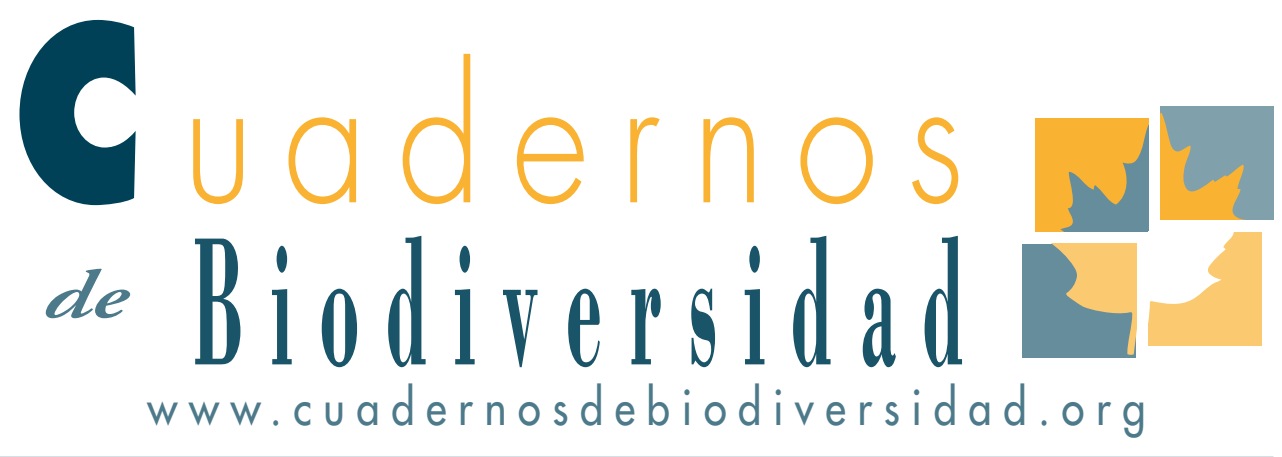

\title{
La determinación del sexo en Cícadas (Cycadales)
}

\section{Iglesias-Andreu ${ }^{1}$, P. Octavio-Aguilar ${ }^{1}, N$. Sánchez-Coello ${ }^{1}, A$. Baldo-Romero ${ }^{1}$ \& J.L.Casas ${ }^{2}$}

i Instituto de Biotecnología y Ecología Aplicada (INBIOTECA), Universidad Veracruzana. Av. de las Culturas Veracruzanas No. io i Col. E. Zapata C.P. 9iogo. Xalapa, Veracruz, MÉxico..

2 Centro Iberoamericano de Biodiversidad, Universidad de Alicante, España.

\section{ABSTRACT}

Plants are organisms that offer unique reproductive systems to study sex determination, in other words, the expression of masculinity or femininity. Within the plant kingdom, the separation of sex has emerged over the evolutionary history in more than one hundred occasions. With each new phylogenetically-independent event there is a new reproductive system which may be similar or completely different from the previous system, so in plants there is not an only one mechanism to explain the dioecism. Cycads are a group of primitive dioecious plants that offers an extraordinary opportunity to study the emergence of dioecism in plant species. Several studies have shown that sex in cycads may be deter- mined by homomorphic or heteromorphic sexual chromosomes; although the possibility of epigenetic factors influencing sex determination in these plants cannot be ruled out. The possibility to anticipate the sex of the cycads would allow us to establish management plans focused to the production of quality seeds in sufficient quantity to meet the demand for these ornamental plants.

\section{KEY WORDS:}

Cycads, dioecism, sexual differentiation, evolution of reproductive systems. 


\section{INTRODUCCIÓN}

Las plantas son organismos que ofrecen sistemas únicos de reproducción a través de los cuales podemos estudiar la determinación del sexo, es decir la expresión de la masculinidad o de la feminidad.

Las cícadas son plantas extraordinarias ya que presentan los sexos masculino y femenino separados, condición conocida como dioicismo. Esto es raro para el reino vegetal ya que solo se da en aproximadamente el 6\% de todas las plantas del planeta (Renner \& Ricklef, 1995). Aunado a esta condición dioica, las cícadas son verdaderos fósiles vivientes, ya que han persistido desde el Paleozoico hasta nuestra época prácticamente sin cambio alguno.

En las cícadas las estructuras reproductivas se originan en la parte apical del tallo, es decir en la zona superior de la planta (Figura 1), lo que nos permite apreciar las diferencias entre sus estructuras reproductivas, también conocidos como conos o estróbilos. Antes de la formación de conos los machos y las hembras son prácticamente idénticos.

\section{LA EVOLUCIÓN DEL DIOICISMO EN PLANTAS}

Dentro del reino vegetal, la separación de sexos ha surgido a lo largo de la evolución en más de 100 ocasiones diferentes (Charlesworth, 2002). Con cada nuevo evento, filogenéticamente independiente, surge un nuevo sistema de reproducción que puede ser similar o completamente distinto a los anteriores, por ello en las plantas no hay un mecanismo general que explique el dioicismo.

En muchas especies vegetales la expresión del sexo tiene una base cromosómica, es decir, hay cromosomas específicos para machos y para hembras, como el sistema XY de los humanos. Cada cromosoma sexual es diferente en forma y contenido, lo que se conoce como heterocromía o heteromorfismo cromosómico. Tal es el caso de Rumex acetosa L. (Acetosilla) y Melandrium rubrum (Weigel) Garcke (Silene), donde se han detectado cromosomas sexuales heteromórficos (Ainsworth, 2000). Sin embargo, la heterocromía en plantas dioicas es más bien rara. Muchas plantas dioicas como la papaya o kiwi no
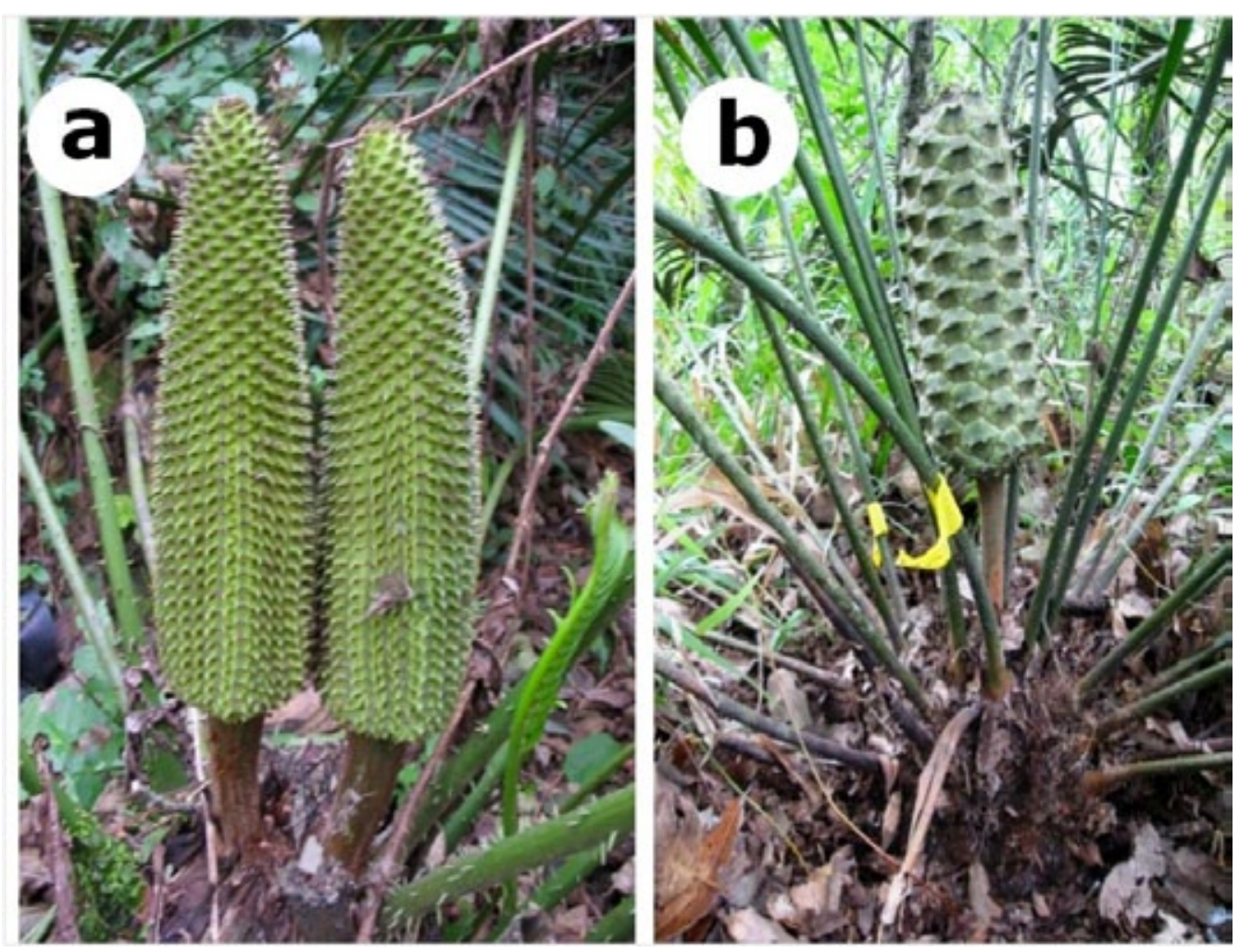

Figura 1. Plantas de Ceratozamia mexicana Brongn.,1846 de la población de Coacoatzintla; Veracruz, México. A) Estróbilo masculino, B) Estróbilo femenino. Fotos: Andrés Rivera-Fernández. 
poseen heteromorfismo cromosómico. En estas especies los genes determinantes del sexo parecen ubicarse en pequeñas regiones de los cromosomas de apariencia normal (Harvey et. al., 1997).

Hoy sabemos que muchos factores ambientales pueden determinar el sexo de las plantas a través de la influencia de fitohormonas, que son componentes químicos secretados por las propias plantas que regulan su crecimiento, floración y supervivencia. Como ejemplos podemos citar a Mercurialis annua (L.) (“Ortiga muerta”, Louis et. al., 1990) y Arisaema triphyllum L. ( "Jack en el púlpito", Policanski, 1981).

Los avances obtenidos en esta dirección han permitido conocer que la expresión del sexo en algunas especies se encuentra bajo un control epigenético que incluye la metilación del ADN o la acetilación de las histonas nucleosómicas. La especie Melandrium album (Mill.) Garcke ha sido un modelo de estudio para la comprensión de la base epigenética del sexo (Janouŝek, 1996). En esta especie se ha encontrado que uno de sus cromosomas $\mathrm{X}$ en las hembras se encuentra hipermetilado para su inactivación. Por ello cuando a los machos se les aplica tratamientos con 5-azacitidina (un compuesto que disminuye la metilación del $\mathrm{ADN}$ ) se ha logrado su conversión a individuos hermafroditas, lo que sugiere una vía probable del surgimiento del diocismo.

De esta forma nos preguntamos ¿Quienes surgieron primero, los machos o las hembras? Como respuesta, el surgimiento de los sexos separados en las plantas tuvo que seguir alguna de estas vías: a) que los machos dieran origen a plantas hermafroditas y después de estas surgieran las hembras, b) que las hembras fueran las que dieron origen a las plantas hermafroditas y después surgieran los machos, c) que fueran plantas hermafroditas las que dieran origen a los otros dos sexos (Gorelick, 2005).

Como ya se mencionó, el dioicismo puede tener orígenes múltiples y diferentes en cada grupo de plantas, por lo que no es posible afirmar que en todos los vegetales la aparición de sexos separados haya seguido solo una de las vías antes mencionadas. Por ello, nos centraremos solo en las cícadas.

\section{EL DIOICISMO EN CÍCADAS}

En Cycas pectinata Buch. Ham se sabe que existen cromosomas sexuales del tipo XY diferenciados por la presencia de pequeñas regiones de $\mathrm{ADN}$ satélite en dos cromosomas somáticos para las hembras, mientras que en los machos solo un cromosoma porta la secuencia satélite (Abraham \& Mathew, 1962). Algo similar ocurre con la cícada Cycas revoluta Thunb. (Segawa et. al., 1971). Sin embargo, en el género mexicano Zamia Miq. no existen satélites asociados a los cromosomas sexuales. Estos resultados evidencian que la determinación del sexo en algunas especies de cícadas está asociada con un tipo de cromosoma sexual XY en el cual el macho es el sexo heterogamético, pero en otros géneros no hay heterocromía. Por eso, algunos autores sugieren que en las cícadas el sexo podría estar asociado con la metilación del ADN (Kanchanaketu et. al., 2007).

Consideremos entonces que para saber el sexo de una cícada, o esperamos que manifieste sus estructuras sexuales (conos masculinos o femeninos) o identificamos la presencia de cromosomas heteromórficos asociados al sexo. El problema radica en que las cícadas se caracterizan por su lento crecimiento y limitada propagación natural (Chávez \& Vovides, 1993) por lo que esperar a que surjan los estróbilos conlleva tener que esperar varios ańos. Por otro lado, no todos los géneros tienen cromosomas heteromóficos.

Según Norstog \& Nicholls (1997), en las especies del género Zamia los eventos reproductivos son escasos y sólo se desarrollan conos en un pequeño porcentaje de los adultos, por lo que las poblaciones producen esporádicamente bajas cantidades de semillas y por ende, una pequeña proporción de plántulas. Además, en muchas cícadas más del 90\% de los individuos mueren antes de llegar a adultos (Octavio-Aguilar et. al., 2008). 
Ante toda esta problemática, sería deseable encontrar la manera de diferenciar sexualmente a los individuos sin tener que esperar a su fase reproductiva. De este modo se podría contribuir a los trabajos de propagación, ya sea convencional o de micropropagación, para la selección de genotipos femeninos que aseguren una mayor producción de conos, tanto para la producción de especies ornamentales o la reintroducción a las poblaciones naturales.

Es por ello que desde hace algunos años el Instituto de Biotecnología y Ecología Aplicada de la Universidad Veracruzana, ha trabajado para conocer la base bioquímica y molecular de la determinación del sexo en algunas especies de cícadas como Ceratozamia mexicana Brongn, Ceratozamia hildae G.P.Landry \& M.C.Wilson y Zamia furfuracea, L.f. in Aiton con un enfoque biotecnológico y ecológico. $\mathrm{Al}$ respecto se han identificado marcadores RAPDs (Iglesias et. al. 2010) que al parecer están ligados a determinantes del sexo masculino. De manera similar se han detectado fragmentos ISSRs asociados al sexo masculino que se están secuenciando para su posible uso en la identificación temprana del sexo en esta especie. Estudios futuros permitirán validar estos resultados y evaluar su posible extensión a otras especies de cícadas con problemáticas similares.

Esta estrategia puede resultar de gran interés no solo para contribuir a la comprensión de los mecanismos involucrados en la diferenciación sexual en plantas, sino también en el orden práctico, ya que nos permitirá emprender acciones encaminadas a restablecer la viabilidad de las poblaciones naturales y establecer planes de aprovechamiento sustentables de estos valiosos recursos.

\section{REFERENCIAS}

Abraham, P. \& Mathew, P.M. 1962. Cytological studies in the Cycads: Sex chromosome in Cycas. Ann. Bot. 26:261-267.

Ainsworth, C. 2000. Boys and girls come out to play: the molecular biology of dioecious plants. Ann. Bot. 86:211221.
Charlesworth, D. 2002. Plant sex determination and sex chromosomes. Heredity. 88:94-101.

Chávez, V.M. \& Vovides, A. P. 1993. Regeneración in vitro de tres especies de Zamia. Boletín Amaranto. 6:12-17.

Gorelick, R. 2005. Environmentally alterable additive genetic effects. Evol. Ecol. Res. 7(3):371-379.

Iglesias, A. L., Luna-Rodríguez, M., Durán-Vázquez, M., Rivera-Fernández, A. \& Sánchez-Coello, N. 2010. Marcadores RAPDs asociados a la expresión del sexo en Ceratozamia mexicana Brongniart (Zamiaceae). RCSCFA. 16(2):139-145.

Harvey, C. F., Gill, G.P. , Fraser , L.G. \& Mcneilage. M.A. 1997. Sex-linked markers and progeny sex ratio in diploid A. chinensis. Sex. Plant Rep. 10:149-154.

Janouŝek, B., Siroky, J. \& Vyskot, B. 1996. Epigenetic control of sexual phenotype in a dioecious plant, Melandrium album. Mol. Genet. 250:483-490.

Kanchanaketu, K., Sangdue, N. Toahsakul, M. \& Hongtrakul, V. 2009. Comparative Karyomorphological Study between Male and Female Plants of Some Cycas and Zamia Species. Nat. Sci. 43:476-485.

Louis, J.P., Augur, C. \& Teller, G. 1990. Cytokinins and differentiation process in Mercurialis annua. Plant Physiol. 94:1535-1541.

Norstog, K.J. \& Nicholls, T.J. 1997. The biology of the Cycads. Cornell University Press, Ithaca, USA.

Octavio-Aguilar, P., González-Astorga, J. \& Vovides, A. P. 2008. Population dynamics of the mexican cycad Dioon edule Lindl. (Zamiaceae): life history stages and management impact. Bot. J. Linn. Soc. 157:381-391.

Policanski, D. 1981. Sex choice and the size advantage in jack-in-the-pulpit (Arisaema triphyllum). Proc. Nat. A. Sci. 78:1306-1308.

Renner, S.S. \& Ricklefs, R.E. 1995. Dioecy and its correlates in the flowering plants. Am. J. Bot. 82:596-606.

Segawa, M., Kishi, S. \& Tatumo, S. 1971. Sex chromosomes of Cycas revoluta. Japan J. Genet. 46(1):33-39. 